\title{
Training of adult bronchoscopy: current state and future prospects
}

\section{Editorial}

Currently, in the United States, the American College of Graduate Medical Education (ACGME) is responsible for the determination of competency of trainees in pulmonary and critical care medicine involving bronchoscopic procedures, entrusted to the fellowship program director. Requirements for determining competency are based on volume according to the ACGME requirements and guidelines published by the American College of Chest Physicians (ACCP), the American Thoracic Society/European Respiratory Society (ATS/ERS), and the British Thoracic Society (BTS). The ACGME's requirements are as the following : fellows must demonstrate knowledge of indications, contraindications, complications, limitations, techniques, and interpretation of results of those diagnostic and therapeutic procedures integral to the discipline, fellows must demonstrate competency in procedural and technical skills, including flexible bronchoscopic procedures where transbronchial and endobronchial biopsies and transbronchial needle aspirations are performed (each fellow must perform a minimum of 100 such procedures), fellows must be able to competently perform all medical, diagnostic, and surgical procedures considered essential for the field of practice, and direct supervision of procedures performed by each fellow must occur until proficiency has been acquired and documented by the program director. The ACCP and ATS/ERS training requirements are as the following: minimum of 20 and 20 rigid bronchoscopic procedures (diagnostic and therapeutic), minimum of 20 and 10 auto fluorescence bronchoscopic procedures, minimum of 50 and 40 endobronchial ultra sound guided procedures, minimum of 25 transbronchial needle aspiration procedure (radial and convex probe, ATS/ERS), minimum of 40 endobronchial ultrasound guided procedures-transbronchial needle aspiration procedures (ATS/ERS), minimum of 15 and 20 LASER procedures, minimum of 15 and 10 electrocautery/argon plasma coagulation procedure, minimum of 10 and 10 cryotherapy procedure, minimum of 5 and 5 brachytherapy procedure, minimum of 10 and 10 phototherapy procedure, minimum of 20 and 10 airway stents silicone (silastic, metallic, hybrid, dynamic Y), minimum of 5 balloon tracheobronchoplasty procedure (ACCP), and minimum of 10 transthoracic needle aspiration procedure (ATS/ERS), respectively.

For many physicians, "see one, do one, teach one" is how we expect to learn. Pulmonary procedures are now more complex with addition of more techniques. The goal for pulmonary fellow's competency requires the learning of a new skill. When teachers get a new class, a new textbook, or change the curriculum to add updated information, they change their lesson plans accordingly. Education begins with the basics, builds upon them, and adds more material as the semester continues. When we get trainees into advanced postgraduate training, we have them jump right into the patient management, often without "teaching" them. We are not trained educators although many of us are clinicians, researchers, and academics. Bronchoscopy education is not as simple as asking "Which way do we push the lever to make the tip of the scope flex?". The art of bronchoscopy is hugely complex. There are seven learning styles taught to educators: aural (auditory),
Volume 2 Issue 6 - 2015

\author{
Attapon Cheepsattayakorn, ${ }^{1,2,3}$ Ruangrong \\ Cheepsattayakorn ${ }^{4}$ \\ 'Editor-in-Chief, Journal of Lung, Pulmonary and Respiratory \\ Research, USA \\ ${ }^{2}$ Oth Zonal Tuberculosis and Chest Disease Center,Thailand \\ ${ }^{3}$ I Oth Office of Disease Prevention and Control, Chiang Mai, \\ Department of Disease Control,Thailand \\ ${ }^{4}$ Department of Pathology, Chiang Mai University, Thailand
}

Correspondence: Attapon Cheepsattayakorn, I0th Zonal Tuberculosis and Chest Disease Center, I 43 Sridornchai Road Changklan Muang Chiang Mai 50100 Thailand, Tel 66-53-140767, 66-53-276364, Fax 66-53-140773, 66-53-273590, Email Attapon 1958@gmail.com; attaponche@yahoo.com

Received: October 6, 2015 | Published: October 6, 2015

visual (spatial), physical (kinesthetic), verbal (linguistic), solitary (intrapersonal), social (interpersonal), and logical (mathematical). All students learn differently. Components of educational method in bronchoscopy include combination of each of these learning styles throughout the process to develop a curriculum for teaching bronchoscopy as most people do not know their ideal learning styles without having to test each trainee for their appropriate learning style. As the educators, we need to stop looking at the number or procedures needed to become competent; this is different for every individual. Developing curricula based upon agreed-upon goals and sound educational standards with optimal kinesthetic and didactic standards are needed for making bronchoscopy education a priority.

\section{Bronchoscopy training and evaluation outside the United States}

Many published articles have indicated that the use of bronchoscopy simulators, web-based learning and endobronchial ultrasonography enhances the learning process. The simulators can evaluate whether individuals have acquired the minimum required skill and knowledge to perform the bronchoscopic procedures. Higher complication rates, greater doses of sedation, and more-prolonged procedures are associated with single apprenticeship-style learning on patients. Lectures should be included in training program and should be complemented with optimal books. Formal demonstration of both virtual and real clinical cases including interactive computer programs will provide the best learning process.

Overall, we advice that : in countries with comparable level of medical care, bronchoscopy training program requirements be 
standardized, simulation specifically be integrated into a structured bronchoscopy teaching curriculum, high-fidelity simulation should be offered in regional simulation centers for accessibility to all training programs, ongoing quality and process improvement systems after skill acquisition be introduced, bronchoscopy training programs incorporate multiple tools, such as hands-on training, casebased reviews, lectures, books, and e-learning, assessment of skill maintenance and improvement in practice be evaluated regularly, professional societies and certifying bodies that oversee training programs for similar procedures in different medical and surgical specialties standardize the requirements where applicable, and professional societies and certifying bodies move from a volumebased certification system to skill acquisition and knowledge-based competency assessment for pulmonary fellows.

\section{Conclusion}

It should be realized that this article is focused on bronchoscopy training in the developed world. We hope that a focus on standardized training content will demonstrate beneficial effects globally. Not just counting the number of procedures performed, but the professional bodies start judging successful skill acquisition with other means. We expect that our advice will be valuable for ongoing bronchoscopy training research and involved procedural training programs.

\section{Acknowledgements}

None.

\section{Conflict of interest}

The author declares no conflict of interest. 